\title{
DIMENSÕES DO SERVIÇO DE REFERÊNCIA VIRTUAL: uma análise do ponto de vista dos usuários
}

\author{
Ieda Pelógia Martins Damian \\ Docente da Universidade de São Paulo, \\ Faculdade de Filosofia, Ciências e Letras \\ de Ribeirão Preto e no Programa de Pós- \\ graduação em Ciência da Informação da \\ Universidade Estadual Paulista Júlio de \\ Mesquita Filho. Doutora em \\ Administração de Organizações pela \\ Universidade de São Paulo. \\ E-mail: iedapm@usp.br
}

\section{Claudio Marcondes de Castro \\ Filho}

Docente da Universidade de São Paulo, Faculdade de Filosofia, Ciências e Letras de Ribeirão Preto. Doutor em Ciência da Informação pela Universidade de São

Paulo.

E-mail: claudiomarcondes@ffclrp.usp.br

\section{RESUMO}

0 serviço de referência virtual diz respeito à disponibilização do serviço de referência por meio de tecnologia de informação, de modo a agregar valor tanto para as bibliotecas quanto para os usuários que interagem por meio desta ferramenta. Para que, no contexto das bibliotecas universitárias, os serviços de referência virtuais tenham seus valores realmente acrescidos, este estudo se propõe a realizar um levantamento dos principais aspectos do serviço de referência virtual do ponto de vista de seus usuários, por meio de análises de ferramentas desenvolvidas e utilizadas em contexto eletrônico. Este levantamento foi realizado junto aos usuários de serviço de referência virtual para, assim, servir de instrumento de avaliação destes serviços. Foi realizado um estudo de caráter bibliográfico e quantitativo, com seleção de amostra não probabilística por conveniência, utilizando como instrumento de coleta de dados um questionário estruturado empregando escala de Likert. Importantes aspectos relacionados a prestação do serviço de referência virtual do ponto de vista de seus usuários puderam ser identificados como a ausência de avaliação constante e de serviços personalizados.

Palavras-chave: Serviço de Referência Virtual. Qualidade do Serviço. Satisfação do Usuário. Dimensões do SRV.

DIMENSIONS OF THE VIRTUAL REFERENCE SERVICE: an analysis from the user's point of view

\section{ABSTRACT}

The Virtual Reference Service concerns the provision of the reference service through information technology in order to add value both for libraries and for users who interact through this tool. So that, in the context of academic libraries, virtual reference services have their values actually increased, this study aims to conduct a survey of the main aspects of virtual reference service from the point of view of its members through tools analysis developed and used in an electronic context. This survey was carried out among virtual reference service users to thus serve assessment tool for these services. A literature review and quantitative study was conducted, 
with selection of non-probabilistic convenience sample, using as a data collection instrument of a structured questionnaire using Likert scale. Important aspects of the provision of virtual reference service from the point of view of its members could be identified as the absence of constant assessment and personalized services.

Keywords: Quality of Service. User Satisfaction. Virtual Reference Service. VRS Dimensions.

\section{INTRODUÇÃO}

A flexibilidade de comunicação, a redução do custo e a troca de grandes quantidades de informação instantaneamente, independentemente da distância geográfica fazem parte de uma série de vantagens oferecidas pela internet (McNEAL et al., 2003). Estes aspectos podem ser utilizados para o oferecimento de Serviço de Referência Virtuais (SRV) de qualidade reconhecida por seus usuários.

O serviço de referência virtual, de acordo com Pereira e Brenha (2011), é tudo o que se faz há séculos no atendimento ao usuário da biblioteca universitária, onde foram agregados valores com as tecnologias que surgiram diante da biblioteconomia moderna.

O objetivo deste estudo é realizar um levantamento dos principais aspectos do SRV do ponto de vista de seus usuários, uma vez que a maioria dos estudos relacionados à análise deste serviço está voltada para os aspectos que auxiliam a avaliação do SRV do ponto de vista de infraestrutura, sem considerar os usuários. Silva e BeuttenMüller (2006) acreditam que estudos sobre o SRV no Brasil são necessários para a consolidação do serviço que fornece a informação solicitada, utilizando os recursos eletrônicos para facilitar na resposta das questões de referência aos usuários.

\section{REFERENCIAL TEÓRICO}

Um levantamento dos principais aspectos a serem considerados na prestação do SRV tornar-se um importante instrumento estratégico que pode sugerir soluções de modo a aperfeiçoar as novas formas de interação com os usuários das bibliotecas universitárias em busca de recursos informacionais. Para tanto, os principais conceitos envolvidos na prestação do SRV são apresentados a seguir. 


\subsection{Serviço de Referência Virtual}

De acordo com a Reference and User Services Association (Rusa, 2015), os serviços de referência são consultas de informação que o pessoal da biblioteca recomenda, interpreta, avalia e/ou utiliza recursos de informação para ajudar os usuários a satisfazerem suas necessidades de informação.

As oportunidades oferecidas pelo meio eletrônico, levaram ao surgimento do SRV, nos Estados Unidos no final da década de 1980 (ARELLANO, 2001). Em 2004, a Rusa definiu o SRV como um serviço de referência iniciado em um contexto eletrônico, usualmente em tempo real, onde os usuários utilizam recursos tecnológicos para se comunicarem com os profissionais de referência, sem estarem fisicamente presentes.

As vantagens do SRV destacadas por Carvalho e Lucas (2005) são as seguintes: as fontes de informação virtual se atualizam rapidamente; demandam menor mão de obra e não necessitam de espaço físico para o armazenamento, e a recuperação da informação torna-se otimizada e precisa. Accart (2012) também menciona os benefícios do SRV como ser acessível em todos os lugares e a todo instante; oferecer, geralmente, serviço gratuito; garantir a proteção dos dados e das informações; orientar para fontes de informação confiáveis; e oferecer um serviço personalizado e de qualidade.

Com o intuito de destacar a importância do desenvolvimento do SRV, Katz (2002) fez uma importante contribuição, especialmente para esta pesquisa, ao afirmar que as bibliotecas deveriam aprender com os sites comerciais maneiras e ferramentas utilizadas para atrair e atender seus usuários. Esta afirmação foi observada e efetiva no desenvolvimento desta pesquisa. E, portanto, o próximo item deste estudo discorre sobre definições e avanços alcançados no campo dos negócios eletrônicos que podem contribuir de maneira efetiva com o desenvolvimento do SRV.

\subsection{Negócios e Serviços Eletrônicos}

Os negócios eletrônicos podem ser definidos, segundo Laudon e Laudon (2010), como a utilização da internet e das tecnologias digitais para executar os processos de negócios de uma organização, que inclui tanto as atividades relacionadas com sua gestão interna como com sua coordenação com fornecedores e parceiros do negócio. 
De acordo com as pesquisas realizadas por Parasuraman, Zeithaml e Berry (1985), os consumidores usam critérios similares para avaliar a qualidade do serviço, independentemente do seu tipo. Os autores alocaram esses critérios em categorias denominadas de "determinantes da qualidade do serviço" que serviram de base para o desenvolvimento de uma escala, denominada ServQual, utilizada para realizar a comparação entre as expectativas dos clientes e suas reais percepções de desempenho.

Hernon e Calvert (2005) desenvolveram um instrumento de pesquisa baseado no ServQual, denominado e-ServQual, voltado para bibliotecas, que se concentra no exame dos serviços de biblioteca on-line e descobriram que, as dimensões mais importantes para os usuários da biblioteca foram facilidade de uso, coleções, confiabilidade, customização/personalização, segurança/privacidade/confiança, apoio, facilidade de acesso, articulação, flexibilidade e estética do site.

As dimensões de serviços on-line, para Yang, Jun e Peterson (2004), centram-se em três dimensões de qualidade: capacidade de resposta, confiabilidade e cortesia.

Parasuraman, Zeithaml e Malhotra (2005) desenvolveram uma escala para medir a qualidade do serviço oferecido por sites, denominada E-S-QUAL, composta pelas dimensões: eficiência, realização, disponibilidade do sistema e privacidade.

Ainda em relação aos avanços alcançados no campo do comércio eletrônico, é possível afirmar que os aspectos relacionados ao marketing podem trazer importantes contribuições para a avaliação do SRV e, portanto, serão agregados ao modelo proposto neste estudo. Deste modo, torna-se oportuno que os conceitos, bem como os instrumentos de mensuração relacionados a este assunto, sejam abordados.

De acordo com Constantinides (2002), as diferenças essenciais entre o contexto físico e o on-line têm levado a reavaliação dos princípios de marketing: o tradicional mix de marketing composto pelos 4 Ps (em inglês product, price, promotion e place) (em português produto, preço, promoção e lugar) tornou-se incompatível com o contexto eletrônico e, então, o autor propôs o mix de marketing para web que identifica os elementos críticos de marketing para tal ambiente, composto pelos 4 Ss: escopo (scope), site, sinergia (synergy) e sistema (system).

Também se faz necessário considerar a experiência na web que, de acordo com Constantinides (2004), diz respeito à impressão que o consumidor tem da empresa online. A impressão do cliente virtual e suas ações são influenciadas pelo design, eventos, emoções, atmosfera e outros elementos experimentados durante a interação com um site. 
Estes elementos foram agrupados em três categorias de fatores que, segundo o autor, influenciam a experiência na web e que são associados ao sucesso ou ao fracasso dos sites que são os fatores de funcionalidade, os psicológicos e os de conteúdo.

Os aspectos influenciadores apresentados podem ser um meio para as organizações analisarem a satisfação de seus usuários. Uma decorrência desse aspecto poderia ser maior utilização do SRV. Nesse sentido, é que se coloca o tópico a seguir, que discute a importância da satisfação dos usuários com este tipo de serviço.

\subsection{Serviço de Referência Virtual: satisfação do usuário}

A satisfação das necessidades de informação dos usuários, de acordo com Carvalho e Lucas (2005), ocorre por meio da antecipação das respostas através da atualidade e variedade de produtos e serviços oferecidos, necessitando para tanto de um estudo prévio de usuário para identificar as necessidades de informação do grupo em questão. As percepções e as necessidades dos clientes são, também para a Rusa (2015), importantes medidas da qualidade que impactam nos serviços de referência.

Para Parker e Mathews (2001), a satisfação é o sentimento resultante do julgamento que o usuário faz da avaliação do que foi recebido em comparação ao que era esperado, ou seja, a satisfação é o resultado da avaliação da percepção da diferença entre as percepções do desempenho e as expectativas.

Para determinar a satisfação do usuário, Nilsen (2006) coloca que é necessário que fatores como a comunicação interpessoal, a qualidade das fontes, a correção das respostas e a eficácia do sucesso do sistema sejam avaliados com frequência. Por meio de um estudo para analisar a satisfação de usuários de serviço de referência presencial e virtual, o autor identificou que o comportamento dos funcionários, links escondidos a esses serviços, solicitação de informações pessoais que não são solicitadas na referência presencial, falta de descrição ou instrução do serviço e barreiras técnicas são os principais obstáculos para a utilização do SRV (NILSEN, 2006).

Para conhecer o grau de satisfação dos utilizadores sobre os serviços prestados pela Biblioteca do Conhecimento Online, Costa (2014) desenvolveu um questionário baseado na escala ServQual e e-ServQual, que levou a obtenção de dados importantes sobre a definição de linhas de ação que devem ser adotadas no futuro. 
Pomerantz (2008) destaca que a avaliação é um componente crítico da gestão de SRV e ressalta ainda que o serviço não existe sem seus usuários, de modo que percepções do atendimento dos usuários são uma parte crítica de qualquer avaliação.

\subsection{Qualidade e Satisfação}

Como as bibliotecas fornecem de serviços, Hernon e Calvert (2005) reforçam a importância de abordar a qualidade do serviço: a interação da biblioteca com seus usuários afeta a qualidade e natureza do serviço prestado, sendo importante procurar atender e/ou superar as expectativas desses usuários que mudam ao longo do tempo e que são moldadas por experiências com outros prestadores de serviços.

Os principais requisitos para a qualidade do serviço de informação destacados por Vergueiro e Carvalho (2001) são: entendimento das necessidades e expectativas dos usuários; segurança, que inclui confiabilidade, cortesia e comunicabilidade; e adoção de linguagem adequada por parte dos profissionais de informação.

O usuário, de acordo com Valls e Vergueiro (2006), é o mais indicado para definir suas necessidades e o nível de qualidade do serviço recebido.

Madu e Madu (2002), com base em uma aprofundada revisão da literatura, propuseram 15 dimensões da qualidade do serviço on-line: desempenho; apresentação; estrutura; estética; confiabilidade; capacidade de armazenamento; manutenção; segurança e integridade do sistema; confiança; capacidade de resposta; diferenciação de produtos e customização; políticas da loja Web; reputação; garantia; e empatia.

Yang, Peterson e Cai (2003) utilizaram 14 dimensões: resposta, credibilidade, facilidade de uso, confiabilidade, conveniência, comunicação, acesso, competência, cortesia, personalização, melhoria contínua, colaboração, segurança/privacidade e estética. Parasuraman, Zeithaml e Malhotra (2005) desenvolveram a escala ES-QUAL, composta de quatro dimensões: eficiência, realização, disponibilidade do sistema e privacidade.

Com o intuito de identificar as dimensões que mais influenciam na qualidade de serviço on-line, Yang, Jun e Peterson (2004) desenvolveram uma pesquisa por meio da qual foi possível identificar a dimensões confiabilidade, capacidade de resposta, competência, facilidade de uso, segurança e portfólio de produtos. 
Hernon e Calvert (2005) concluíram que, apesar da exploração em torno da qualidade do serviço (e-SQ), ainda não existia um consenso sobre as dimensões a serem exploradas. Por meio de revisão da literatura e de entrevistas com grupos focais, os autores chegaram a 10 dimensões relacionadas a esses serviços: facilidade de uso; estética do site; link; coleções; confiabilidade; suporte; segurança/privacidade/ confiança; facilidade de acesso; flexibilidade; e personalização/customização.

Loiacono, Watson e Goodhue (2007) desenvolveram um instrumento para a avaliação de consumidores de sites denominado WebQual composto por 12 dimensões: tarefa informativa, informações adaptadas, confiança, tempo de resposta, facilidade de compreensão, operações intuitivas, apelo visual, capacidade de inovação, apelo emocional, imagem consistente, completude on-line e vantagem relativa.

Outro aspecto norteador utilizado no desenvolvimento deste estudo foi a afirmação de Yang, Jun e Peterson (2004) de que nem todos os atributos de qualidade de serviço têm o mesmo impacto sobre as percepções dos consumidores de serviços on-line. Assim, a identificação dos fatores que mais contribuem para a percepção de qualidade destes serviços precisa ser desenvolvida.

Pomerantz e Luo (2006) desenvolveram um estudo para avaliar a eficácia do SRV via chat no atendimento às necessidades de informação dos usuários e concluíram que avaliar os serviços de referência da perspectiva do usuário constitui uma solução para medir o valor e a utilidade das informações fornecidas por tal serviço.

Com o objetivo de explorar maneiras de atenuar a lacuna entre a qualidade do SRV e seu baixo consumo em bibliotecas universitárias, $\mathrm{Mu}$ (2011) desenvolveu uma extensa pesquisa onde verificou que a chave do sucesso do SRV é ir além do próprio serviço de referência virtual, ou seja, é preciso preencher a lacuna entre o usuário e o bibliotecário, ao invés de entre o usuário e o serviço.

Estas pesquisas que foram detalhadas serviram de base para a construção do modelo a ser proposto neste estudo conforme discorre o próximo item deste trabalho.

\subsection{Dimensões dos Serviços de Referência Virtuais}

Inicialmente, todos os aspectos citados pelos autores nos itens anteriores deste trabalho foram agrupados em dimensões. Ao final desta etapa, foram obtidas sete 
dimensões: Acesso; Capacidade de Resposta; Comunicação; Confiabilidade; Estímulo; Produto; e Segurança, conforme descrito na Tabela um.

Tabela 1 - Dimensões do Modelo Proposto

\begin{tabular}{|c|c|}
\hline Dimensão & Autores \\
\hline Acesso & $\begin{array}{l}\text { Parasuraman, Zeithaml e Berry (1985), Madu e Madu (2002); } \\
\text { Yang, Peterson e Cai (2003), Constantinides (2004); } \\
\text { Parasuraman, Zeithaml e Malhotra (2005), Hernon e Calvert } \\
\text { (2005), Nilsen (2006), Pomerantz e Luo (2006); Loiacono, } \\
\text { Watson e Goodhue (2007), Costa (2014) }\end{array}$ \\
\hline $\begin{array}{l}\text { Capacidade de } \\
\text { Resposta }\end{array}$ & $\begin{array}{l}\text { Parasuraman, Zeithaml e Berry (1985), Vergueiro e Carvalho } \\
\text { (2001), Madu e Madu (2002); Yang, Jun, e Peterson (2004); } \\
\text { Parasuraman, Zeithaml e Malhotra (2005); Nilsen (2006); } \\
\text { Loiacono, Watson e Goodhue (2007), Connaway, Radford e } \\
\text { Dickey (2008) }\end{array}$ \\
\hline Comunicação & $\begin{array}{l}\text { Parasuraman, Zeithaml e Berry (1985), Vergueiro e Carvalho } \\
\text { (2001); Constantinides (2002), Madu e Madu (2002); Yang, } \\
\text { Peterson e Cai (2003), Yang, Jun, e Peterson (2004); } \\
\text { Constantinides (2004), Parasuraman, Zeithaml e Malhotra } \\
\text { (2005), Hernon e Calvert (2005); Nilsen (2006); Pomerantz e } \\
\text { Luo (2006); Loiacono, Watson e Goodhue (2007); Mu (2011) }\end{array}$ \\
\hline Confiabilidade & $\begin{array}{l}\text { Parasuraman, Zeithaml e Berry (1985), Vergueiro e Carvalho } \\
\text { (2001), Madu e Madu (2002), Yang, Peterson e Cai (2003), } \\
\text { Constantinides (2004), Yang, Jun, e Peterson (2004), Hernon e } \\
\text { Calvert (2005), Loiacono, Watson e Goodhue (2007), Connaway, } \\
\text { Radford e Dickey (2008), Costa (2014) }\end{array}$ \\
\hline Estímulo & $\begin{array}{l}\text { Constantinides (2002); Madu e Madu (2002), Yang, Peterson e } \\
\text { Cai (2003); Constantinides (2004), Hernon e Calvert (2005), } \\
\text { Nilsen (2006); Loiacono, Watson e Goodhue (2007) }\end{array}$ \\
\hline Produto & $\begin{array}{l}\text { Yang, Peterson e Cai (2003), Constantinides (2004), Yang, Jun e } \\
\text { Peterson (2004), Hernon e Calvert (2005), Nilsen (2006); } \\
\text { Loiacono, Watson e Goodhue (2007), Costa (2014) }\end{array}$ \\
\hline Segurança & $\begin{array}{l}\text { Parasuraman, Zeithaml e Berry (1985), Vergueiro e Carvalho } \\
\text { (2001), Madu e Madu (2002), Yang, Peterson e Cai (2003), Yang, } \\
\text { Jun e Peterson (2004), Hernon e Calvert (2005), Parasuraman, } \\
\text { Zeithaml e Malhotra (2005), Nilsen (2006), Costa (2014) }\end{array}$ \\
\hline
\end{tabular}

Fonte: elaborado pelos autores

Por meio do embasamento teórico que levou a elaboração das dimensões apresentadas na tabela 1, foram identificados os aspectos pertinentes a este estudo que devem ser avaliados em cada dimensão, visualizados no quadro um. 
Quadro 1 - Aspectos Avaliados

\begin{tabular}{|c|c|}
\hline DIMENSÃO & ASPECTOS AVALIADOS \\
\hline Acesso & $\begin{array}{l}\text { sempre encontro os materiais eletrônicos que necessito. } \\
\text { os links funcionam. } \\
\text { é fácil navegar pelo site. } \\
\text { facilmente localizo os serviços disponibilizados. } \\
\text { disponho de site bem estruturado, com menus que me ajudam a } \\
\text { compreender como informação é organizada. }\end{array}$ \\
\hline $\begin{array}{l}\text { Capacidade de } \\
\text { Resposta }\end{array}$ & $\begin{array}{l}\text { meus problemas são resolvidos rapidamente. } \\
\text { minhas questões são respondidas rapidamente. } \\
\text { os funcionários prestam assistência especializada quando preciso. }\end{array}$ \\
\hline Comunicação & $\begin{array}{l}\text { a interação ocorre de maneira cortês. } \\
\text { posso entrar em contato com os bibliotecários a qualquer momento. } \\
\text { a comunicação através de bate-papo na internet ou de mensagens de } \\
\text { texto é eficaz. } \\
\text { forneço feedback sobre a minha satisfação com o atendimento recebido. }\end{array}$ \\
\hline Confiabilidade & $\begin{array}{l}\text { percebo que a biblioteca fornece uma melhoria contínua no } \\
\text { atendimento ao usuário. } \\
\text { tenho ajuda e suporte técnico. } \\
\text { minhas estratégias de busca são registradas e posso usá-las novamente. } \\
\text { o site funciona bem. }\end{array}$ \\
\hline Estímulo & $\begin{array}{l}\text { economizo tempo. } \\
\text { posso acessá-los nos horários que me são mais convenientes. } \\
\text { posso fazer download de materiais rapidamente. } \\
\text { estes serviços são amplamente divulgados. } \\
\text { Eu pretendo utilizar esses serviços novamente. }\end{array}$ \\
\hline Produto & $\begin{array}{l}\text { tenho acesso a uma gama de recursos na minha área específica. } \\
\text { tenho acesso a conteúdos eletrônicos atualizados. } \\
\text { recebo alerta sobre o material recém-publicado com base em um perfil } \\
\text { de usuário personalizado. } \\
\text { tenho acesso as informações on-line claras e de fácil compreensão. }\end{array}$ \\
\hline Segurança & $\begin{array}{l}\text { o site é seguro. } \\
\text { minhas transações são precisamente registradas. } \\
\text { me agrada manter o anonimato. }\end{array}$ \\
\hline
\end{tabular}

Fonte: elaborado pelos autores

Conforme pode ser observado no quadro 1 , o que se pretende analisar diz respeito aos aspectos funcionais da avaliação de serviço de referência virtual, envolvendo elementos da qualidade do serviço, do mix de marketing e da experiência web do ponto de vista do usuário.

\section{METODOLOGIA}

Foi realizado um estudo de caráter bibliográfico com o objetivo de ampliar os conhecimentos sobre os conceitos trabalhados, onde foram consultados trabalhos disponibilizados por entidades acadêmicas nacionais e internacionais, governamentais e 
não governamentais, bases de dados científicas. Gil (2007) destaca a relevância do levantamento bibliográfico ao afirmar que este permite ao pesquisador cobrir uma gama de fenômenos muito mais ampla do que aquela que poderia pesquisar diretamente.

Foi realizada uma pesquisa quantitativa, com seleção de amostra não probabilística por conveniência, o que possibilitou aos pesquisadores uma maior compreensão do fenômeno, ressalvado o aspecto de que os resultados obtidos apresentam restrições a uma generalização ampla. A análise quantitativa utilizada neste estudo pode ser definida, segundo Richardson (2004), como um método que faz uso de parâmetros quantificáveis, tanto para a coleta dos dados quanto para o tratamento desses dados, e se desenvolve por meio da utilização de técnicas estatísticas.

O instrumento utilizado para coletar os dados nesta etapa da pesquisa foi um questionário estruturado para todos os respondentes já que, como afirmou Richardson (2004), este instrumento enquanto técnica de pesquisa cumpre as funções de descrever as características e medir determinadas variáveis de um grupo social.

Os questionários estruturados, compostos por 29 questões fechadas com escala de Likert de cinco pontos, foram enviados, por meio eletrônico, a usuários de serviço de referência virtuais de bibliotecas universitárias públicas e privadas. Para aumentar a taxa de retorno dos questionários enviados por meio de correio eletrônico e ajudar uma instituição, um valor simbólico de $\mathrm{R} \$ 0,30$ foi doado ao Grupo de Apoio a Criança com Câncer para cada questionário respondido. Esta doação foi fundamentada em Synodinos (2003) que afirmou que os incentivos podem aumentar a taxa de resposta.

A primeira parte do questionário foi construída com o objetivo de caracterizar o entrevistado. Para a elaboração das demais afirmações do questionário, foram utilizados os aspectos relacionados às dimensões que compõem o modelo proposto para analisar a qualidade dos serviços de referência virtuais.

\section{RESULTADOS}

O convite para responder ao questionário e contribuir com a pesquisa foi desenvolvido e enviado pela internet para cerca de 30.000 e-mails de alunos, pesquisadores, docentes e funcionários de instituições de ensino superior. Foram obtidas 1.487 respostas, ou seja, 4,96\% dos e-mails enviados retornaram. Não houve necessidade de descartar nenhuma resposta por algum problema de preenchimento. 
Para aumentar a confiabilidade da pesquisa e o número de questionários preenchidos corretamente, o convite continha, além da informação sobre a doação a uma entidade, os nomes das organizações relacionadas diretamente a esta pesquisa, ou seja, USP (Universidade de São Paulo) e do GACC (Grupo de Apoio a Criança com câncer). A amostra foi obtida via questionário disponibilizado por meio de link constante no convite, durante os meses de março e abril de 2015.

Os dados coletados nesta etapa foram inseridos no software de análise estatística SPSS (statistical package for social sciences) para a aplicação de técnicas estatísticas multivariadas.

Quadro 2 - Atributos avaliados positivamente

\begin{tabular}{|l|l|l|}
\hline \multicolumn{1}{|c|}{ Dimensões } & \multicolumn{1}{|c|}{ Questões } & \multicolumn{1}{|c|}{$\begin{array}{c}\text { Soma das } \\
\text { Notas }\end{array}$} \\
\hline Estímulo & $\begin{array}{l}\text { Posso acessá-los nos horários que me são mais } \\
\text { convenientes. }\end{array}$ & 6819 \\
\hline Estímulo & Economizo tempo. & 6208 \\
\hline Estímulo & Eu pretendo utilizar esses serviços novamente. & 6152 \\
\hline Segurança & O site é seguro. & 5826 \\
\hline Produto & Tenho acesso a conteúdos eletrônicos atualizados. & 5814 \\
\hline
\end{tabular}

Fonte: elaborada pelos autores

Conforme os dados apresentados no quadro 2, verificou-se que das seis dimensões utilizadas neste estudo, três têm seus atributos dentre os mais bem avaliados, que são as dimensões Estímulo, Segurança e Produto. Vale destacar que dos cinco aspectos mais bem avaliados, três compõem a dimensão Estímulo, demonstrando que os aspectos e recursos disponíveis para estimular a utilização do meio eletrônico por parte dos usuários dos serviços de referência virtuais estão produzindo efeitos positivos.

Quadro 3 - Atributos avaliados negativamente

\begin{tabular}{|l|l|l|}
\hline Dimensões & \multicolumn{1}{|c|}{ Questões } & $\begin{array}{c}\text { Soma das } \\
\text { Notas }\end{array}$ \\
\hline Confiabilidade & Tenho ajuda e suporte técnico. & 4438 \\
\hline Comunicação & $\begin{array}{l}\text { A comunicação através de bate-papo na internet ou de } \\
\text { mensagens de texto é eficaz. }\end{array}$ & 4275 \\
\hline Produto & $\begin{array}{l}\text { Recebo alerta sobre o material recém-publicado com } \\
\text { base em um perfil de usuário personalizado. }\end{array}$ & 3843 \\
\hline Estímulo & Estes serviços são amplamente divulgados. & 3763 \\
\hline Comunicação & $\begin{array}{l}\text { Forneço feedback sobre a minha satisfação com o } \\
\text { atendimento recebido. }\end{array}$ & 3664 \\
\hline
\end{tabular}

Fonte: elaborada pelos autores 
Em relação às questões com as menores pontuações atribuídas pelos usuários do SRV, conforme os dados demonstrados no quadro 3, quatro das seis dimensões utilizadas neste estudo estão presentes: Confiabilidade, Comunicação, Produto e Estímulo. Dos cinco aspectos com as menores pontuações, dois compõem a dimensão Comunicação. Esta constatação demonstra que os usuários atribuem grande importância a comunicação usuário-bibliotecário e que, portanto, representa aspectos que devem receber atenção especial por parte dos gestores do SRV.

Apesar do destaque dado aos atributos com maiores e menores pontuações, uma observação muito importante é que dos 28 atributos avaliados, apenas um ("Forneço feedback sobre a minha satisfação com o atendimento recebido"), não obteve superior à média de 2,5, ainda assim, ficando muito próximo a ela, com a pontuação de 2,46.

A partir das médias das pontuações alcançadas é possível concluir que, de modo geral, os usuários do SRV se mostraram satisfeitos com os atributos pesquisados.

A segurança oferecida por um site é fator crítico de sucesso discutido por importantes autores como Parasuraman, Zeithaml e Berry (1985), que a tem como um de seus "determinantes da qualidade do serviço"; Vergueiro e Carvalho (2001) que consideraram a segurança como um dos principais requisitos para a qualidade de um serviço de informação; Constantinides (2004) que coloca a confiança on-line como aspecto influenciador da experiência na web; Hernon e Calvert (2005) que abordaram em suas pesquisas aspectos de segurança como privacidade e confiança; Parasuraman, Zeithaml e Malhotra (2005) que, desenvolveram o modelo E-S-QUAL, composto também pela dimensão Privacidade, que verifica em que grau o site é seguro e protege as informações do cliente; e Accart (2012) que discorre sobre a proteção dos dados.

De acordo com a pesquisa quantitativa realizada neste estudo é possível concluir que os sites realmente têm se preocupado em oferecer um ambiente seguro aos seus usuários porque o atributo relacionado à segurança do site estava entre os cinco atributos que obtiveram, na pesquisa quantitativa, as maiores pontuações.

Para que o SRV possa ser implantado com sucesso, autores como Silva e BeuttenMüller (2006); e Connaway, Radford e Dickey (2008) ressaltaram a importância de desenvolver estratégias de marketing adequadas para que os usuários possam tomar conhecimento das ofertas de SRV e, assim, além de utiliza-los, promovê-los a potenciais usuários. Para os autores, estas estratégias ocorrem de modo insuficiente. 0 atributo 
relacionado à divulgação do SRV estava entre os atributos que receberam as piores pontuações na pesquisa, ressaltando a carência da divulgação destes serviços.

A comunicação on-line é um atributo importante destacado por vários autores, entre os quais estão Parasuraman, Zeithaml e Berry (1985); e Constantinides (2004). As vantagens oferecidas pela comunicação por meio da utilização do correio eletrônico e do chat foram também muito ressaltadas, de modo especial por Pessoa e Cunha (2007). 0 fato da afirmação sobre a eficácia da comunicação através de bate-papo na internet ou de mensagens de texto estar entre as afirmações que receberam as piores pontuações na pesquisa é algo que deve chamar atenção, demonstrando que, apesar de sua importância, é um atributo que ainda deve ser aprimorado.

Pomerantz e Luo (2006) destacaram a importância de explorar os feedbacks dos usuários que utilizam os serviços. Vergueiro e Carvalho (2001) também fizeram importantes considerações sobre a avaliação do usuário sobre o serviço. Apesar da importância destacada dos feedbacks dos usuários, os resultados alcançados na pesquisa demonstram que solicitar e fazer uso de feedbacks de usuários parece ser algo que não deve acontecer com frequência, uma vez que o atributo relacionado ao fornecimento de feedbacks estava entre os que receberam as piores pontuações.

\section{CONSIDERAÇÕES FINAIS}

Com o objetivo de realizar um levantamento dos principais aspectos do SRV do ponto de vista de seus usuários, optou-se por uma pesquisa quantitativa por meio de aplicação de questionários em meio eletrônico. Com a realização deste levantamento, importantes pontos que devem auxiliar as bibliotecas universitárias, sejam elas públicas ou privadas, foram destacados:

- $\quad$ A necessidade de avaliar: o SRV com mais frequência por meio da solicitação de feedbacks aos seus usuários;

- $\quad$ A falta de estratégias de marketing para a adequada divulgação do SRV;

- $\quad$ A carência de serviços personalizados.

As principais contribuições deste estudo para a área acadêmica estão relacionadas com a identificação de aspectos importantes que permitem analisar os serviços de 
referência virtuais do ponto de vista de seus usuários, como também, suscitar a necessidade de novos estudos acerca dos aspectos levantados.

Em relação à área gerencial, é possível afirmar que este estudo contribuiu com o destaque de aspectos importantes para analisar os serviços de referência virtuais sob a ótica dos usuários destes serviços, auxiliando-os a identificar elementos fundamentais para os usuários destes serviços que poderiam não estar recebendo a devida atenção de seus gestores, bem como auxiliar em todo o processo decisório.

Sendo o SRV um assunto dinâmico e multidisciplinar, seria impossível, em um único estudo, cobrir de maneira eficiente em todos os seus aspectos. Portanto, o estudo em questão teve seu foco nos serviços prestados aos usuários, ciente de que outros elementos devem ser estudados em pesquisas futuras.

\section{AGRADECIMENTOS}

Os autores agradecem à FAPESP pelos auxílios ao desenvolvimento dessa pesquisa.

\section{REFERÊNCIAS}

ACCART, J-P. Serviço de referência: do presencial ao virtual. Brasília: Briquet de Lemos/ Livros, 2012.

ARELLANO, M. A. M. Serviços de Referência Virtual, Ciência da Informação, Brasília, v. 30, n. 2, p. 7-15, maio/ago. 2001.

CARVALHO, L. S.; LUCAS, E. R. O. Serviço de Referência e Informação: do tradicional ao on-line. In: ENCONTRO NACIONAL DE CIÊNCIA DA INFORMAÇÃO, Salvador. Anais... Salvador: ENANCIB, 2005.

CONNAWAY, L. S.; RADFORD, M. L.; DICKEY, T. J. On the Trail of the Elusive Non-User: What Research in Virtual Reference Environments Reveals, Bulletin of the American Society for Information Science and Technology, v. 34, n. 2, Special Section, Virtual Reference Services, 2008.

CONSTANTINIDES, E. The 4S Web-Marketing Mix model, Electronic Commerce Research and Applications, v. 1, p. 57-76, 2002.

. Influencing the online consumer's behavior: the Web experience. Internet Research, v. 14, n. 2, p. 111-126, 2004.

COSTA, M. T. Biblioteca do Conhecimento Online: pela construção da Sociedade do Conhecimento. 2014. Disponível em:

<http://www.apbad.pt/Downloads/congresso9/COM3_.pdf>. Acesso em: 06 maio 2014.

GIL, A. C. Como elaborar projetos de pesquisa. 4. ed. São Paulo: Atlas, 2007. 
HERNON, P., CALVERT, P. E-service quality in libraries: Exploring its features and dimensions, Library \& Information Science Research, n. 27, p. 377-404, 2005.

KATZ, B. Digital Reference, The Reference Librarian, v. 38, n. 79-80, p. 1-17, 2002.

LAUDON, K. C.; LAUDON, J. P. Sistemas de informação gerenciais. Tradução Luciana do Amaral Teixeira. Revisão Técnica Belmiro Nascimento João. 9. ed. São Paulo: Pearson Prentice Hall, 2010.

LOIACONO, E. T.; WATSON, R. T.; GOODHUE, D. L. WebQual: An Instrument for Consumer Evaluation of Web Sites. International Journal of Electronic Commerce, Spring, v. 11, n. 3, p. 51-87, 2007.

MADU, C. N.; MADU, A. A. Dimensions of e-quality, International Journal of Quality \& Reliability Management, v. 19, n. 3, p. 24 6-258, 2002.

McNEAL, R. S., TOLBERT, C. J., MOSSBERGER, K., DOTTERWEICH, L. J., Innovating in Digital Government in the American States, Social Science Quarterly, v. 84, n. 1, p. 52-70, 2003.

MU, X. et al. A Survey and Empirical Study of Virtual Reference Service in Academic Libraries, The Journal of Academic Librarianship, v. 37, n. 2, p. 120-129, 2011.

NILSEN, K. Comparing users' perspectives of in-person and virtual reference, New Library World, v. 107, n. 1222/1223, p. 91-104, 2006.

PARASURAMAN, A.; ZEITHAML, V. A.; BERRY, L. L. A Conceptual Model of Service Quality and Its Implications for Future Research, Journal of Marketing, v. 49, n. 4, p. 41-50, 1985.

PARASURAMAN, A.; ZEITHAML, V. A.; MALHOTRA, A. E-S-QUAL A Multiple-Item Scale for Assessing Electronic Service Quality, Journal of Service Research, v. 7, n. 3, p. 213-233, 2005.

PARKER, C., MATHEWS, B. P. Customer satisfaction: contrasting academic and consumers' interpretations, Marketing Intelligence \& Planning, v. 19, n. 1, p. 38-44, 2001.

PEREIRA, M. R. S.; BRENHA, T. C. P. Serviço de Referência em Bibliotecas: reflexões. In: Congresso Brasileiro de Biblioteconomia, Documentação e Ciência da Informação, 24, Maceió.

Anais... Maceió: FEBAB, 2011.

PESSOA, P.; CUNHA, M. B. Perspectivas dos Serviços de Referência Digital, Informação \& Sociedade: Estudos, João Pessoa, v. 17, n. 3, p. 69-82, set./dez. 2007.

POMERANTZ, J. LUO, L. Motivations and uses: Evaluating virtual reference service from the users' perspective, Library \& Information Science Research, v. 28, p. 350-373, 2006.

POMERANTZ, J. Virtual reference services: Evaluation of online reference services, Bul. Am. Soc. Info. Sci. Tech., v. 34, p. 15-19, 2008.

RICHARDSON, R. J. Metodologia da pesquisa aplicável às ciências sociais. In: Beuren, Ilse Maria (org). Como elaborar trabalhos monográficos em contabilidade: teoria e prática. 2. ed. São Paulo: Atlas, 2004.

RUSA. Reference and User Services Association. Guidelines for Implementing and Maintaining Virtual Reference Services, 2004. 
Measuring and Assessing Reference Services and Resources: A Guide. 2015.

Disponível em: <http://www.ala.org/rusa/sections/rss/rsssection/rsscomm/ evaluationofref/measrefguide>. Acesso em: 23 fev. 2015.

SILVA, A. K. A.; BEUTTENMÜLLER, Z. F. O serviço de referência online nas bibliotecas virtuais da região nordeste, Pesquisa Brasileira em Ciência da Informação e Biblioteconomia, v. 1, n. 1, 2006.

SYNODINOS, N.E. The "art" of questionnaire construction: some important considerations for manufacturing studies, Integrated Manufacturing Systems, v. 14, n.3, p. 221-237, 2003.

VALLS, V. M.; VERGUEIRO, W. C. S. A gestão da qualidade em serviços de informação no Brasil: uma nova revisão de literatura, de 1997 a 2006, Perspectivas em Ciência da Informação, Belo Horizonte, v. 11 n. 1, p. 118-137, jan./abr. 2006.

VERGUEIRO, W.; CARVALHO, T. de. Definição de indicadores de qualidade: a visão dos administradores e clientes de bibliotecas universitárias, Perspectivas em Ciência da Informação, Belo Horizonte, v. 6, n. 1, p. 27-40, 2001.

YANG, Z.; JUN, M.; PETERSON, R. T. Measuring customer perceived online service quality: Scale development and managerial implications, International Journal of Operations \& Production Management, v. 24, n. 11, p. 1149-1174, 2004.

YANG, Z.; PETERSON, R. T.; CAI, S. Services quality dimensions of Internet retailing: an exploratory analysis, Journal of Services Marketing, v. 17, n. 7, p. 685-700, 2003. 